\title{
Unusual Case of a Pseudoaneurysm in a Replaced Common Hepatic Artery Arising from Superior Mesenteric Artery
}

\author{
Karthik Marpalli Vasudeva ${ }^{1}$, Ashwin S Polnaya ${ }^{2}$, Sandhya Rao $\mathrm{K}^{3}$ \\ ${ }^{1}$ Assistant Professor, Department of Radiodiagnosis, A.J. Institute of Medical Sciences and Research Centre, Mangalore, \\ Karnataka, ${ }^{2}$ Associate Professor, Department of Radiodiagnosis, A.J. Institute of Medical Sciences and Research Centre, \\ Mangalore, Karnataka, ${ }^{3}$ Junior Resident, Department of Radiodiagnosis, A.J. Institute of Medical Sciences and Research \\ Centre, Mangalore, Karnataka, India.
}

Corresponding author: Karthik Marpalli Vasudeva, Assistant Professor, Department of Radiodiagnosis, A.J. Institute of Medical Sciences and Research Centre, Mangalore, Karnataka, India

DOI: http://dx.doi.org/10.21276/ijcmsr.2019.4.4.37

How to cite this article: Karthik Marpalli Vasudeva, Ashwin S Polnaya, Sandhya Rao KUnusual Case of a pseudoaneurysm in a replaced common hepatic artery arising from superior mesenteric artery. . International Journal of Contemporary Medicine Surgery and Radiology. 2019;4(4):D158-D159.

\section{A B S T R A C T}

Introduction: Vascular complication following biliary surgery is life threatening. Knowledge of variant vascular anatomy is a pre requisite for successful treatment. We report a rare case of a pseudoaneurysm in a replaced common hepatic artery arising from superior mesenteric artery successfully treated with coil embolisation.

Case report: An elderly patient who was a known hypertensive and diabetic with past history of biliary stenting and surgical biliary exploration presented with melena and blood in abdominal drain since 3 days. An emergency CT scan revealed a pseudoaneurysm in the replaced common hepatic artery originating from the superior mesenteric artery. The artery with the pseudoaneurysm was coil embolised and the patient was discharged in stable condition with no active evidence of bleeding.

Conclusion: Common hepatic artery can originate from superior mesenteric artery and can get injured during biliary surgery. Performing CT Angiography of the abdomen will lead to a quick and prompt diagnosis . Coil embolisation of the pseudoaneurysm is the first line of management in such cases.

Keywords: Common Hepatic Artery, Pseudoaneurysm, Coil Embolisation.

\section{INTRODUCTION}

Hepatic artery variations are quite common in literature. However Common hepatic artery originating from the superior mesenteric artery is rare. It can get injured during hepatobiliary and gastrointestinal surgeries leading to formation of a pseudoaneurysm. Preoperative knowledge of variant anatomy is thus a necessity. Computed Tomography (CT) Angiography is the modality of choice to diagnose pseudoaneurysm. Coil embolisation is the treatment of choice for pseudoaneurysm.

\section{CASE REPORT}

69 year old Male patient who is a known hypertensive, diabetic with past history of biliary stenting and surgical biliary exploration for impacted bile duct stones presented with intermittent bleeding per rectum and blood in abdominal drain since 3 days. Patient was hypotensive on evaluation and he was medically resuscitated with fluids and blood transfusion. He underwent computed tomography angiogram evaluation which revealed an arterial phase contrast blush suggestive of pseudoaneurysm arising from the common hepatic artery. The common hepatic artery was originating from superior mesenteric artery (Figure 1).
He further underwent a conventional angiogram which confirmed the same (Figure 2a). An injection of cefoperazone sulbactum 1.5 gram intra venously every 12 th hourly was started. Mesenteric angiography with coil embolisation of the replaced common hepatic artery pseudoaneurysm distal to the gastroduodenal artery (Figure 2b) was done. Patient

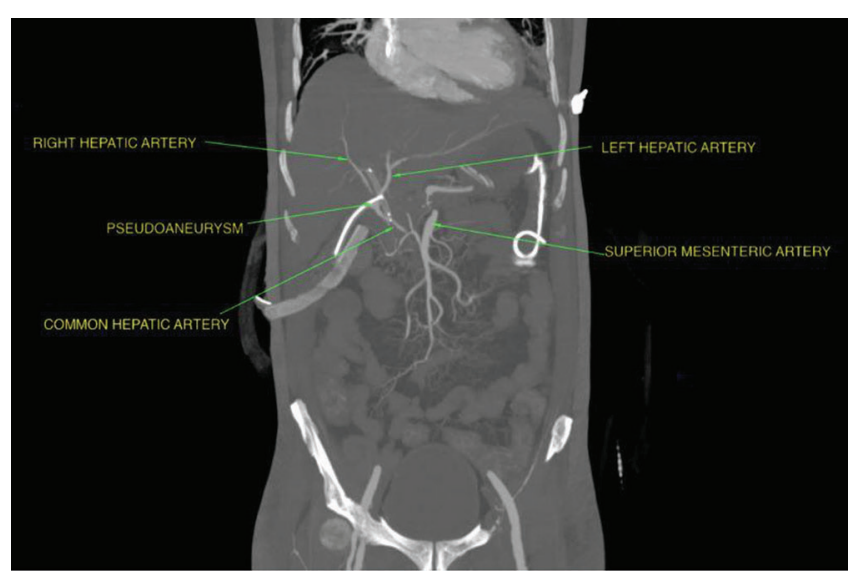

Figure-1: CT angiography of abdomen - coronal view showing pseudoaneurysm in common hepatic artery arising from superior mesenteric artery. 

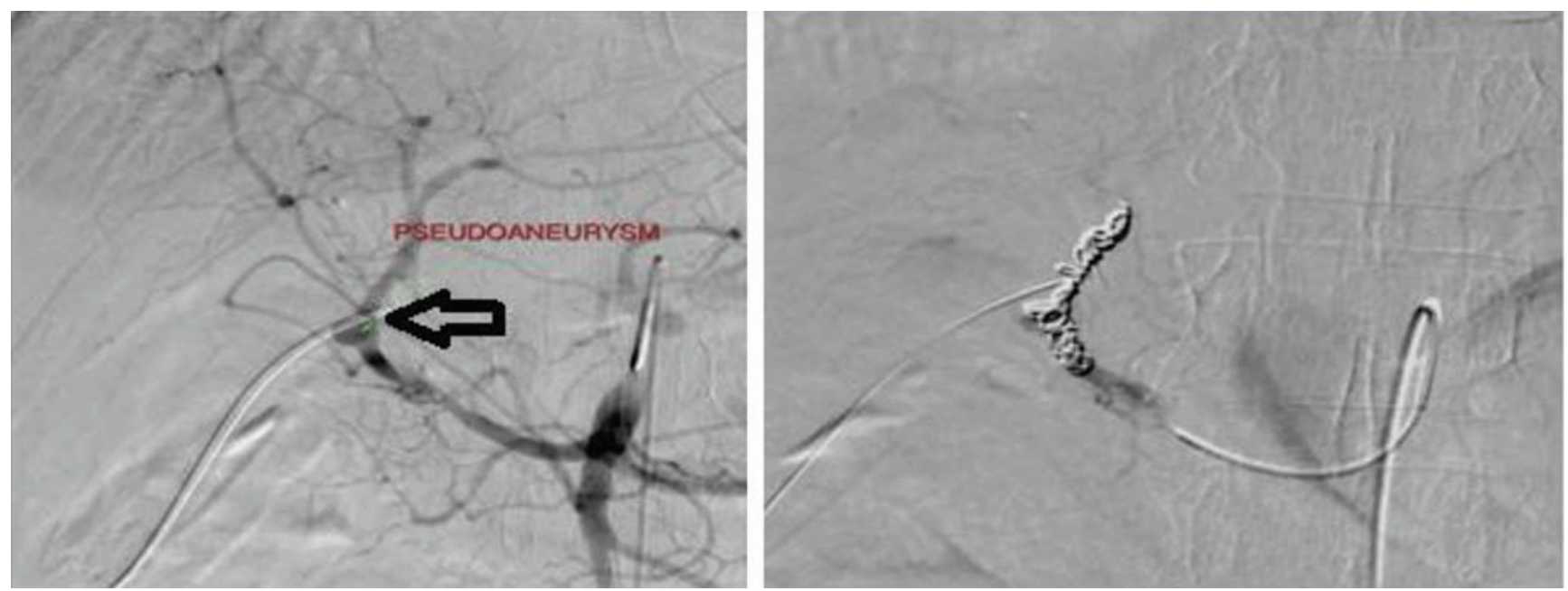

Figure-2A: Digital substraction angiography - pre embolisation showing the pseudoaneurysm in Replaced common hepatic artery. Figure-2B: Digital substraction angiography -post embolisation of Replaced common hepatic artery showing coils used for occluding the artery.

was discharged in stable condition with no active evidence of bleeding.

\section{DISCUSSION}

Vascular complications following biliary surgeries are rare. ${ }^{1}$ They usually present with hemobilia and melena. Most of the vascular complications in the laprascopic cholecystectomy involve the right hepatic artery. ${ }^{2,3}$ common hepatic artery injury following biliary surgeries are rare. A replaced common hepatic artery arising from superior mesenteric artery is a rare anatomical variation with incidence ranging from $0.4 \%$ to $4.5 \% .{ }^{4}$ It is known as hepaticomesenteric trunk.

In our case replaced common hepatic artery is arising from superior mesenteric artery from its posterior aspect. It is coursing between pancreas and inferior vena cava for a short segment and is then directed anteriorly in the pancreaticoduodenal groove before bifurcating into right and left hepatic artery. Common hepatic artery is in close relation to common bile duct in its distal segment.

Injury to the replaced common hepatic artery originating from superior mesenteric artery and presenting as pseudoaneurysm is infrequent in literature. The pseudoaneurysm in our case was arising just before bifurcation of the common hepatic artery in the hepatic artery proper. The gastroduodenal artery was proximal to the aneurysm.

The Initial investigation of choice to diagnose pseudoaneurym is computed tomography angiography. However catheter angiography remains the gold standard. ${ }^{5}$ The first line management in hepatic artery pseudoaneurysms is Trans arterial coil embolisation. ${ }^{2}$ If there is failure of coil embolisation surgery must be done. In our case we used four angiographic coils to successfully block the hepatic artery proper segment of the replaced common hepatic artery distal to the gastroduodenal artery.

\section{CONCLUSION}

Common hepatic artery can originate from superior mesenteric artery and can get injured during biliary surgery. CT Angiography of the abdomen will lead to a quick and prompt diagnosis . Coil embolisation of the pseudoaneurysm is the first line management in such cases.

\section{REFERENCES}

1. Davidoff, A.M., Pappas, T.N., Murray, E.A., Hillern, D.J., Johnson, R.D., and Baker, M.E. Mechanisms of major biliary injury during laparoscopic cholecystectomy. Ann Surg. 1992; 215(2): 196-202

2. T. W. J. Lennard, S. M. Plusa, J. L. R. Forsythe, and D. L. Richardson,. Treatment of right hepatic artery injury by percutaneous embolisation. The Lancet, 1994;344(8932):1306-1307.

3. T. Bulut, S. Yamaner, D. Bugra, A. Akyuz, K. Acarli, and A. Poyanli. False aneurysm of the hepatic artery after laparoscopic cholecystectomy. Acta Chirurgica Belgica, 2002;102(6):459-463,

4. Noussios G, Dimitriou I, Chatzis I, Katsourakis A. The Main Anatomic Variations of the Hepatic Artery and Their Importance in Surgical Practice: Review of the Literature. J Clin Med Res. 2017; 9(4):248-252.

5. Tulsyan N, Kashyap VS, Greenberg RK, Sarac TP, Clair DG, Pierce G, et al. The endovascular management of visceral artery aneurysms and pseudoaneurysms. J Vasc Surg. 2007; 45(6):276-83.

\section{Source of Support: Nil; Conflict of Interest: None}

Submitted: 26-10-2019; Accepted: 16-11-2019; Published online: 25-12-2019 